\title{
A rigidity theorem for Lagrangian deformations
}

\author{
Mauricio D. Garay
}

\begin{abstract}
We consider deformations of singular Lagrangian varieties in symplectic manifolds. We prove that a Lagrangian deformation of a Lagrangian complete intersection is analytically rigid provided that this is the case infinitesimally. This result is given as a consequence of the coherence of the direct image sheaves of relative infinitesimal Lagrangian deformations.
\end{abstract}

\section{Introduction}

We investigate local deformations of singular Lagrangian complete intersections in a symplectic manifold. These Lagrange varieties appear in microlocal analysis as characteristic varieties of quantum integrable systems (see, e.g., [CP94, CP99, DS99]). Deformations of the ideal generated by micro-differential equations induce deformations of the corresponding Lagrangian varieties and, if we regard as equivalent micro-differential ideals which are conjugated by a Fourier integral operator, then equivalent Lagrangian varieties are Lagrangian varieties which are isomorphic up to a symplectic change of coordinates.

Following previous investigations of Pham (see, e.g., [Pha00]), Colin de Verdière observed that in the semi-classical limit, the map from the versal deformation space of the micro-differential ideal to that of its characteristic variety is an isomorphism [Col03] (see also [Gar05b]). He used this correspondence to prove a formal microlocal versal deformation theorem for one-dimensional microdifferential equations. In the case when the characteristic variety is a monomial deformation of a quasi-homogeneous Lagrangian curve singularity, Colin de Verdière proved that the equivalence could be obtained by convergent series. Then, he posed the problem of the existence of a Lagrangian versal deformation for general Lagrangian curve germs. In [Gar04], we showed that this result was a consequence of a slight modification of Brieskorn's coherence theorem [Bri70], similar results were obtained in [Lan95, KL93].

In this paper, we investigate the higher-dimensional problem. We will use the case of plane curves as a guiding example. Our main result is that infinitesimally Lagrangian versal deformations are rigid, i.e., such a Lagrangian deformation admits only trivial deformations (given by a change of coordinates which preserves the symplectic form). This result is weaker than the statement 'infinitesimal Lagrangian versality' implies versality. Nevertheless, it is very likely that a more refined analysis in the spirit of [Pou74] actually leads to this result. Moreover, our result is sufficient to prove a Mather type result for stable integrable systems [Gar05a].

As a simple application of the rigidity theorem, we consider the Lagrangian variety germ $(L, 0)$ given by the equations $p_{i} q_{i}=0, i=1, \ldots, n$. In that case, the rigidity theorem implies that the $n$-parameter deformation $p_{i} q_{i}=\varepsilon_{i}$ is rigid. That infinitesimal rigidity implies rigidity in this example was stated in [NP91] without proof.

Received 28 May 2004, accepted in final form 18 November 2004

2000 Mathematics Subject Classification 70H06, 32S65.

Keywords: normal form theory, symplectic geometry, integrable systems, Lagrangian varieties.

This work was supported by the Deutsche Forschungsgemeinschaft (Project no. SPP 1094 and Forschungsstipendium GA 786/1-1).

This journal is (C) Foundation Compositio Mathematica 2005. 


\section{A RIGIDITY THEOREM FOR LAGRANGIAN DEFORMATIONS}

Our strategy can be summarized as follows. If the deformation module of some deformation theory is of finite type and if it is compatible with base changes then infinitesimal versal deformations are rigid. If, moreover the sum of deformations is defined then infinitesimally versal deformation are indeed versal. This paper is only a concrete illustration of these facts; it is organized as follows.

We start the first section by adapting to the relative case the deformation theory considered by Sevenheck and van Straten for Lagrangian varieties [SvS03, Sev03]. The resulting Lagrangian versal deformation space was, in fact, already described by Du'c Nguyen and Pham in [NP91]. Then we state the main algebraic result namely the finiteness of the deformation module. We postpone the proof of this statement to $\S 4$ since this is only a variant of the proof given by Sevenheck and van Straten in the absolute case [SvS03]. This result was used in our joint paper [GS03] with van Straten.

In $\S 2$, we state the rigidity theorem. We consider only a naive viewpoint of versality namely versality over a smooth base. In $\S 3$, the proof of the rigidity theorem is given. In $\S 4$, we state and prove the coherence of the direct image sheaves of the relative Lagrange complex.

The results of this paper can easily be adapted for real analytic Lagrangian varieties. The formal aspect of the theory of Lagrangian deformations is treated in Sevenheck's thesis [Sev03]. Deformations of compact complex Lagrangian submanifolds of Kähler manifolds were considered in [Voi92]. An earlier version of this paper was pre-published in [Gar02].

\section{Lagrangian deformations}

\subsection{Deformations of real compact Lagrangian manifolds}

Let us consider the vector space $\mathbb{R}^{2 n}=\{(x, y)\}$ together with the symplectic structure

$$
\omega=\sum_{i=1}^{n} d x_{i} \wedge d y_{i} .
$$

Recall that a Lagrangian manifold in $\mathbb{R}^{2 n}$ is an $n$-dimensional submanifold of $\mathbb{R}^{2 n}$ on which the symplectic form $\omega$ vanishes.

To understand the construction of the Lagrange complex, it is useful to investigate the deformations of a smooth compact Lagrangian submanifold $L \subset \mathbb{R}^{2 n}$. For such a manifold, the DarbouxWeinstein theorem asserts that there exists a symplectomorphism

$$
\varphi: \mathbb{R}^{2 n} \longrightarrow T^{*} L
$$

which maps a tubular neighbourhood of $L \subset \mathbb{R}^{2 n}$ to a tubular neighbourhood of the zero section in the cotangent bundle $T^{*} L$ to $L$ (see [Wei73]).

Via the map $\varphi$, a small one parameter deformation $\left(L_{t}\right)$ of $L$ is mapped to the family of graphs of a one parameter family of maps

$$
\alpha_{t}: L \longrightarrow T^{*} L
$$

that is, to a family of differential one forms.

It is readily verified that $L_{t}$ is Lagrangian if the one-form $\alpha_{t}$ is closed and that $L_{t}$ is Hamiltonian isotopic to $L_{0}=L$ if $\alpha_{t}$ is exact.

Consequently, the space of $C^{\infty}$-small deformations of $L$ over some base $\Lambda$ modulo Hamiltonian isotopies is parameterized by the maps from $\Lambda$ to the first de Rham cohomology group $H^{1}(L, \mathbb{R})$ of $L$ sending $0 \in \Lambda$ to $0 \in H^{1}(L, \mathbb{R})$. 


\subsection{The relative Lagrange complex}

We consider now the complex holomorphic situation, that is, a complex manifold $M$ of dimension $2 n$ together with a holomorphic symplectic two-form $\omega \in \Omega_{M}^{2}(M)$.

The Poisson bracket $\{f, g\}$ of two holomorphic functions $f, g \in \mathcal{O}_{M}(U)$ is defined by the formula

$$
\{f, g\} \omega^{n}=d f \wedge d g \wedge \omega^{n-1} .
$$

Recall that a Lagrangian submanifold of $M$ is an $n$-dimensional holomorphic manifold on which the symplectic form vanishes. By Lagrangian variety $L \subset M$, we mean a reduced complex space of pure dimension $n$ defined by an ideal sheaf $\mathcal{I}_{L}$ which is closed under the Poisson bracket. This means that

$$
\{f, g\} \in \mathcal{I}_{L}(U) \quad \text { whenever } f, g \in \mathcal{I}_{L}(U)
$$

for any open subset $U \subset M$. Over the smooth locus of $L$, it is readily seen that both definitions agree.

We consider now the situation with parameters. Let $\Lambda$ be a complex manifold (the parameter space) with a marked point, denoted by 0 , on it. The Poisson bracket on $M$ lifts to an $\mathcal{O}_{\Lambda}$-linear Poisson bracket on $\Lambda \times M$. We shall say that a variety

$$
L \subset(\Lambda \times M)
$$

is a Lagrange variety if its projects one-to-one to a Lagrange variety of $M$. Let $Z \subset(\Lambda \times M)$ be a reduced complex subspace with ideal sheaf $\mathcal{I}$. The sequence

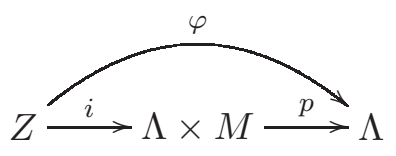

where $i$ is the inclusion and $p$ is the projection, is called a Lagrangian deformation of $\varphi^{-1}(0)$ if $\varphi=p \circ i$ is a flat deformation and if the fibres of $\varphi$ are reduced Lagrangian varieties.

For brevity, we will simply write $\varphi: Z \longrightarrow \Lambda$ for a given Lagrangian deformation.

For a given $f=f_{1} \wedge \cdots \wedge f_{k} \in \Lambda^{k} \mathcal{I} / \mathcal{I}^{2}$, we denote by $f^{j} \in \bigwedge^{k-1} \mathcal{I} / \mathcal{I}^{2}$ the element

$$
f^{j}=(-1)^{j} f_{1} \wedge \cdots \wedge \hat{f}_{j} \wedge \cdots \wedge f_{k} .
$$

The element $\left(f^{i}\right)^{j} \in \bigwedge^{k-2} \mathcal{I} / \mathcal{I}^{2}$ is denoted by $f^{i, j}$.

Definition. The relative Lagrange complex, denoted $\left(\mathcal{C}_{Z / \Lambda}, \delta\right)$, of the Lagrangian deformation

$$
\varphi: Z \longrightarrow \Lambda
$$

is the complex of sheaves on $Z$ defined by

$$
\mathcal{C}_{Z / \Lambda}^{k}=\mathcal{H o m}_{\mathcal{O}_{Z}}\left(\bigwedge^{k} \mathcal{I} / \mathcal{I}^{2}, \mathcal{O}_{Z}\right)
$$

and the $k$ th differential $\delta: \mathcal{C}_{Z / \Lambda}^{k} \longrightarrow \mathcal{C}_{Z / \Lambda}^{k+1}$ is given by

$$
\delta[\varphi](f)=\sum_{1 \leqslant i \leqslant n}\left\{f_{i}, \varphi\left(f^{i}\right)\right\}-\sum_{1 \leqslant i<j \leqslant n} \varphi\left(\left\{f_{i}, f_{j}\right\} \wedge f^{i, j}\right) .
$$

Analogous definitions can be given for germs of Lagrangian deformations.

Notation. If $L$ is a Lagrange variety then the Lagrange complex of $L$, denoted by $\mathcal{C}_{L}$ (which is defined in [SvS03]) is the relative Lagrange complex of the constant deformation $\varphi: L \longrightarrow\{0\}$ (here $Z=L, \Lambda=\{0\}$ ).

Remark. The differentials of the complex $\mathcal{C}_{Z / \Lambda}$ are $\varphi^{-1} \mathcal{O}_{\Lambda}$-linear; therefore its cohomology spaces are $\varphi^{-1} \mathcal{O}_{\Lambda}$-modules. 


\section{A RIGIDITY THEOREM FOR LAGRANGIAN DEFORMATIONS}

Example 1. Denote by $X_{f}$ the Hamilton vector field of a function $f \in \mathcal{O}_{Z}$. Assume that the fibres of $\varphi$ are smooth. Then the evaluation map

$$
\Omega_{Z}^{1} \longrightarrow \mathcal{C}_{Z / \Lambda}^{1}, \quad \alpha \mapsto\left[f \mapsto \alpha . X_{f}\right]
$$

induces an isomorphism between the relative Lagrange complex $\mathcal{C}_{Z / \Lambda}^{\bullet}$ and the relative de Rham complex $\Omega_{Z / \Lambda}^{\circ}$

Example 2. Consider a map germ $\varphi:\left(\mathbb{C}^{2}, 0\right) \longrightarrow(\mathbb{C}, 0)$ with an isolated critical point at 0 . Then the stalk at the origin of the Lagrange complex has only two terms both isomorphic to $\mathcal{O}_{\mathbb{C}^{2}, 0}$ and the differential is given by $h \mapsto\{h, \varphi\}$. Therefore, the map $\mathcal{O}_{\mathbb{C}^{2}, 0} \longrightarrow \Omega_{\mathbb{C}^{2}, 0}^{2}, m \mapsto m \omega$ maps the module $H^{1}\left(\mathcal{C}_{Z / \Lambda, 0}^{\cdot}\right)$ to its Brieskorn lattice $\Omega_{\mathbb{C}^{2}, 0}^{2} / d \varphi \wedge d \mathcal{O}_{\mathbb{C}^{2}, 0}$. (Here and in the following, given a sheaf $\mathcal{F}$ we denote by $\mathcal{F}_{0}$ its fibre at 0 .)

Example 3. Take $n=2$ and assume that the ideal of $(L, 0)$ is generated by two commuting function germs $f_{1}, f_{2}$. Then the complex has three terms respectively isomorphic to $\mathcal{O}_{L, 0}, \mathcal{O}_{L, 0}^{2}, \mathcal{O}_{L, 0}$. The differentials are respectively given by $\delta h=\left(\left\{h, f_{1}\right\},\left\{h, f_{2}\right\}\right)$ and $\delta\left(m_{1}, m_{2}\right)=\left\{m_{1}, f_{2}\right\}+\left\{f_{1}, m_{2}\right\}$.

\subsection{Equivalence of Lagrangian deformations}

Definition. A Lagrangian deformation germ $\varphi^{\prime}:\left(Z^{\prime}, 0\right) \longrightarrow\left(\Lambda^{\prime}, 0\right)$ is called $\mathcal{L}$-induced (respectively, $\mathcal{L}$-equivalent) from (respectively, to) $\varphi:(Z, 0) \longrightarrow(\Lambda, 0)$ if there exists a commutative diagram

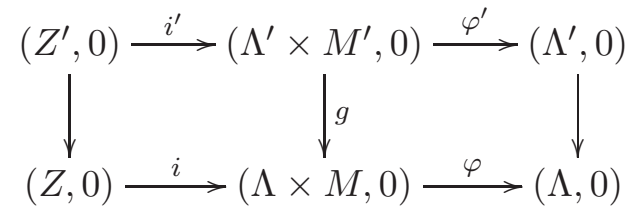

where $i^{\prime}, i$ denote the inclusions and $g$ is a Poisson mapping germ (respectively, a biholomorphic Poisson mapping germ).

Definition. A Lagrangian deformation germ $\varphi:(Z, 0) \longrightarrow(\Lambda, 0)$ is called rigid if any deformation germ $\varphi^{\prime}:\left(Z^{\prime}, 0\right) \longrightarrow(\Lambda \times \mathbb{C}, 0)$ such that the restriction of $\varphi^{\prime}$ above $\Lambda \times\{0\}$ equals $\varphi$, is induced from $\varphi$.

\section{A deformation germ}

$$
\varphi:(Z, 0) \longrightarrow(\Lambda, 0),
$$

of a Lagrangian variety germ $(L, 0)$ is called $\mathcal{L}$-versal if any deformation of $(L, 0)$ over any smooth basis is induced from $\varphi$.

It is readily verified that the first cohomology space of the complex $\mathcal{C}_{L, 0}$ is equal to the $\mathbb{C}$-vector space of first-order Lagrangian deformations of $(L, 0)$ modulo infinitesimally trivial deformations, where the coordinate changes have to be symplectic.

The ideal of $Z$ at the origin is generated by holomorphic function germs

$$
F_{1}, \ldots, F_{p}:\left(\mathbb{C}^{k} \times \mathbb{C}^{2 n}, 0\right) \longrightarrow(\mathbb{C}, 0), \quad(\Lambda \times M, 0) \approx\left(\mathbb{C}^{k} \times \mathbb{C}^{2 n}, 0\right) .
$$

We sometimes use the notation $F=\left(F_{1}, \ldots, F_{p}\right)$ for the deformation germ $\varphi:(Z, 0) \longrightarrow(\Lambda, 0)$. 


\section{D. GARAY}

\subsection{The Lagrangian Kodaira-Spencer map}

We keep the same notations but we assume now that $Z$ is a complete intersection. In this case, we get isomorphisms

$$
\mathcal{C}_{Z / \Lambda, 0}^{k} \longrightarrow \bigwedge^{k} \mathcal{O}_{Z, 0}, \quad \varphi \longmapsto \sum_{i_{1}<\cdots<i_{k}} \varphi\left(F_{i_{1}} \wedge \cdots \wedge F_{i_{k}}\right) e_{i_{1}} \wedge \cdots \wedge e_{i_{k}},
$$

where $\left\{e_{1}, \ldots, e_{n}\right\}$ is the canonical basis of the vector space $\mathbb{C}^{n}$, that is, $e_{1}=(1,0, \ldots, 0)$, $e_{2}=(0,1,0, \ldots, 0), \ldots, e_{n}=(0, \ldots, 0,1)$.

We define a Kodaira-Spencer mapping (or rather the stalk at the origin of a Kodaira-Spencer mapping) by

$$
\begin{aligned}
\theta_{F}: T_{0} \Lambda & \longrightarrow H^{1}\left(\mathcal{C}_{Z / \Lambda, 0}\right) \\
v & \longmapsto[(D F . v)], \quad(\Lambda, 0) \approx\left(\mathbb{C}^{k}, 0\right) .
\end{aligned}
$$

Here $T_{0} \Lambda$ denotes the tangent space to $\Lambda$ at the origin.

For instance, choose local coordinates $\lambda_{1}, \ldots, \lambda_{k}$ at the origin in $\Lambda$. Then the image of the vector $\partial_{\lambda_{i}} \in T_{0} \Lambda$ is given by

$$
\theta_{F}\left(\partial_{\lambda_{i}}\right)=\left[\left(\partial_{\lambda_{i}} F_{1}, \ldots, \partial_{\lambda_{i}} F_{n}\right)\right] .
$$

This Kodaira-Spencer map is well-defined, indeed differentiating the following equality along $v$

$$
\left\{F_{j}, F_{k}\right\}=\sum_{i=1}^{n} a_{i} F_{i}
$$

we get that

$$
\left\{d F_{j} \cdot v, F_{k}\right\}+\left\{F_{j}, d F_{k} \cdot v\right\}=\sum_{i=1}^{n}\left(d a_{i} \cdot v\right) F_{i}+\sum_{i=1}^{n} a_{i}\left(d F_{i} \cdot v\right) .
$$

Thus, the cocycle DF.v $\in \mathcal{C}_{Z / \Lambda, 0}^{1}$ is a coboundary.

Definition. The map $\theta_{F}: T_{0} \Lambda \longrightarrow H^{1}\left(\mathcal{C}_{Z / \Lambda, 0}^{\bullet}\right)$ defined above is called the relative Lagrangian Kodaira-Spencer map of $F$.

As $Z$ is a complete intersection, the restriction to $\lambda=0$ gives a surjective mapping from $\mathcal{C}_{Z / \Lambda}^{k}$ to $\mathcal{C}_{L}^{k}$. Thus, we get an absolute Kodaira-Spencer mapping:

$$
\begin{aligned}
\bar{\theta}_{F}: T_{0} \Lambda & \longrightarrow H^{1}\left(\mathcal{C}_{L, 0}\right) \\
v & \longmapsto\left[(D F . v)_{\mid \lambda=0}\right]
\end{aligned}
$$

and the following commutative diagram.

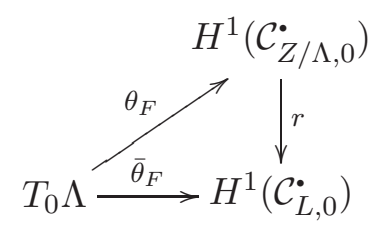

Here $r$ is the restriction to $\lambda=0$. To get a clear picture of this diagram, let us denote by $\mathcal{M}$ the maximal ideal of the local ring $\mathcal{O}_{\Lambda, 0}$ and by $\mathcal{M} H^{1}\left(\mathcal{C}_{Z / \Lambda, 0}^{\bullet}\right)$ the image of the multiplication mapping

$$
\mathcal{M} \otimes \mathcal{O}_{\Lambda, 0} H^{1}\left(\mathcal{C}_{Z / \Lambda, 0}^{\bullet}\right) \longrightarrow H^{1}\left(\mathcal{C}_{Z / \Lambda, 0}^{\bullet}\right)
$$

Proposition 1. The kernel of the map $r$ defined above is equal to $\mathcal{M} H^{1}\left(\mathcal{C}_{Z / \Lambda, 0}^{\bullet}\right)$. 


\section{A RIGIDITY THEOREM FOR LAGRANGIAN DEFORMATIONS}

Proof. The vector space $\mathcal{M} H^{1}\left(\mathcal{C}_{Z / \Lambda, 0}^{\bullet}\right)$ is obviously contained in the kernel of $r$. We show that the induced map

$$
\bar{r}: H^{1}\left(\mathcal{C}_{Z / \Lambda, 0}^{\bullet}\right) / \mathcal{M} H^{1}\left(\mathcal{C}_{Z / \Lambda, 0}^{\bullet}\right) \longrightarrow H^{1}\left(\mathcal{C}_{L, 0}^{\bullet}\right)
$$

is injective. Let us denote by

$$
\varphi_{p}: Z_{p} \longrightarrow \Lambda_{p}, \quad p=0, \ldots, k
$$

the restriction of $\varphi: Z \longrightarrow \Lambda$ above the $\mathbb{C}$-vector subspace

$$
\Lambda_{p}=\left\{\lambda \in \Lambda: \lambda_{1}=\cdots=\lambda_{p}=0\right\}, \quad \Lambda_{0}=\Lambda,
$$

where $\lambda_{1}, \ldots, \lambda_{k}$ denote local coordinates at the origin in $\Lambda$. We have exact sequences of complexes

$$
0 \longrightarrow \mathcal{C}_{Z_{p} / \Lambda_{p}, 0} \longrightarrow \mathcal{C}_{Z_{p} / \Lambda_{p}, 0} \longrightarrow \mathcal{C}_{Z_{p+1} / \Lambda_{p+1}, 0} \longrightarrow 0
$$

where $0 \leqslant p<k$.

These exact sequences induce long exact sequences in cohomology.

$$
\cdots \longrightarrow H^{k}\left(\mathcal{C}_{Z_{p} / \Lambda_{p}, 0}\right) \longrightarrow H^{k}\left(\mathcal{C}_{Z_{p+1} / \Lambda_{p+1}, 0}\right) \longrightarrow H^{k+1}\left(\mathcal{C}_{Z_{p} / \Lambda_{p}, 0}\right) \longrightarrow \cdots
$$

It is readily seen that the $\mathcal{O}_{\Lambda_{p}, 0}$-module $H^{0}\left(\mathcal{C}_{Z_{p} / \Lambda_{p}, 0}\right)$ can be identified with the module $\varphi_{p}^{-1}\left(\mathcal{O}_{\Lambda_{p}, 0}\right)$. Thus, in the exact sequence the map

$$
H^{0}\left(\mathcal{C}_{Z_{p} / \Lambda_{p}, 0}\right) \longrightarrow H^{0}\left(\mathcal{C}_{Z_{p+1} / \Lambda_{p+1}, 0}\right)
$$

is surjective and, therefore, the exact sequence splits. This shows that the induced maps

$$
\frac{H^{1}\left(\mathcal{C}_{Z_{p} / \Lambda_{p}, 0}^{\bullet}\right)}{\lambda_{p+1} H^{1}\left(\mathcal{C}_{Z_{p} / \Lambda_{p}, 0}^{\bullet}\right)} \longrightarrow H^{1}\left(\mathcal{C}_{Z_{p+1} / \Lambda_{p+1}, 0}\right)
$$

are injective. Finally, the injectivity of these maps implies in turn that the map $\bar{r}$ is injective. This proves the proposition.

Remark 1. If the absolute Kodaira-Spencer mapping $\bar{\theta}_{F}$ is surjective, then the proposition implies the exact sequence

$$
0 \longrightarrow \mathcal{M} H^{1}\left(\mathcal{C}_{Z / \Lambda, 0}^{\bullet}\right) \stackrel{i}{\longrightarrow} H^{1}\left(\mathcal{C}_{Z / \Lambda, 0}^{\bullet}\right) \stackrel{r}{\longrightarrow} H^{1}\left(\mathcal{C}_{L, 0}^{\bullet}\right) \longrightarrow 0
$$

where $i$ denotes the inclusion.

\subsection{The finiteness theorem}

Following [SvS03], we introduce a class of singularities which plays the role of isolated singularities in symplectic geometry. On the total space $Z$ of the deformation

$$
\varphi: Z \longrightarrow \Lambda
$$

of a Lagrangian variety $L \subset M$ a stratification is defined as follows. Let $f_{1}, \ldots, f_{k}$ be generators of the ideal of $Z$ at a point $x$. Denote by $V_{x}$ the $\mathbb{C}$-vector space generated by Hamilton vectorfields of $f_{1}, \ldots, f_{k}$ evaluated at $x$. For each $j$, the stratum $Z_{j}$ is defined by the condition

$$
x \in Z_{j} \Longleftrightarrow \operatorname{dim} V_{x}=j .
$$

We have $Z=\bigcup_{j=0}^{n} Z_{j}$ where $2 n=\operatorname{dim}(M)$.

The following notion was introduced in [SvS03] in the absolute case where it is called 'condition $(\mathrm{P})$ '.

Definition. A Lagrangian deformation $\varphi: Z \longrightarrow \Lambda$ is called pyramidal if for any $k$ the variety $Z_{k}$ is of relative dimension at most $k$. 
Proposition 2. The germ of a pyramidal deformation $\varphi: Z \longrightarrow \Lambda$ at a point $x \in Z_{k}$ is $\mathcal{L}$-equivalent to a deformation germ of the type

$$
\varphi^{\prime}:\left(Z^{\prime} \times \mathbb{C}^{k}, 0\right) \longrightarrow(\Lambda, 0), Z^{\prime} \subset \mathbb{C}^{2 n-2 k}, \quad \mathbb{C}^{k} \subset \mathbb{C}^{2 k}
$$

which is constant on the second factor. Moreover, this decomposition induces a quasi-isomorphism between the complexes $\mathcal{C}_{Z / \Lambda, x}$ and $\mathcal{C}_{Z^{\prime} / \Lambda, 0}$.

The proof of this proposition is straightforward, it is based on a simple symplectic reduction argument (in the absolute case see, e.g., [SvS03]).

Theorem 1 [SvS03]. If $(L, 0)$ is the germ of a pyramidal Lagrangian variety then the cohomology spaces $H^{k}\left(\mathcal{C}_{L, 0}^{*}\right)$ are finite-dimensional vector spaces.

On the basis of examples, we conjecture that the converse to this theorem holds.

Conjecture. If $H^{1}\left(\mathcal{C}_{L, 0}^{\bullet}\right)$ is a finite-dimensional vector space and if $(L, 0)$ is a complete intersection then $(L, 0)$ is pyramidal.

We will prove the following generalisation of the previous theorem.

Theorem 2. If $\varphi:(Z, 0) \longrightarrow(\Lambda, 0)$ is the germ of a pyramidal Lagrangian deformation then the cohomology spaces $H^{k}\left(\mathcal{C}_{Z / \Lambda, 0}\right)$ are $\mathcal{O}_{\Lambda, 0}$-modules of finite type.

In the case $\operatorname{dim} L=1$, the theorem is a slight generalisation of a result due to Brieskorn ([Bri70, Satz 1.1], see also [Gre75] for a more general statement).

The proof of this theorem repeats that of Sevenheck and van Straten in the absolute case. We postpone it until $\S 4$. This result was used in [GS03] in order to prove that $H^{1}\left(\mathcal{C}_{Z / \Lambda, 0}^{*}\right)$ is actually a free $\mathcal{O}_{\Lambda, 0}$ module provided that $\varphi$ is an infinitesimally versal Lagrangian deformation.

\section{The rigidity theorem}

Theorem 3. Let $F:\left(\mathbb{C}^{k} \times \mathbb{C}^{2 n}, 0\right) \longrightarrow\left(\mathbb{C}^{n}, 0\right)$ be a Lagrangian deformation of a Lagrangian complete intersection germ $(L, 0) \subset\left(\mathbb{C}^{2 n}, 0\right)$. Assume that:

(1) $(L, 0)$ is pyramidal;

(2) the absolute Kodaira-Spencer map

$$
\bar{\theta}_{F}: T_{0} \mathbb{C}^{k} \longrightarrow H^{1}\left(\mathcal{C}_{L, 0}^{\bullet}\right)
$$

associated to $F$ is surjective.

Then, the Lagrangian deformation $F$ is rigid.

Example 4. Consider the germ at the origin of the Lagrangian variety

$$
L=\left\{(q, p) \in \mathbb{C}^{2 n}: q_{1} p_{1}=q_{2} p_{2}=\cdots=q_{n} p_{n}=0\right\}
$$

and let $F=\left(F_{1}, \ldots, F_{n}\right)$ be the $n$-parameter deformation defined by

$$
F_{i}=q_{i} p_{i}+\lambda_{i}
$$

The Lagrangian Kodaira-Spencer mapping maps $\partial_{\lambda_{1}}, \ldots, \partial_{\lambda_{n}}$ to the cohomology classes

$$
[(1,0, \ldots, 0)], \ldots,[(0, \ldots, 0,1)] \text {. }
$$

A straightforward computation shows that they generate $H^{1}\left(\mathcal{C}_{L, 0}^{\bullet}\right)$.

Thus, the Lagrangian deformation $F$ is rigid. That infinitesimal Lagrangian versality implies Lagrangian rigidity in this example was assumed without proof in [NP91, § 1.2] (compare [Rus64, Vey78]). 


\section{A RIGIDITY THEOREM FOR LAGRANGIAN DEFORMATIONS}

Corollary 1. A Lagrangian deformation $F:\left(\mathbb{C}^{k} \times \mathbb{C}^{2}, 0\right) \longrightarrow(\mathbb{C}, 0)$ of a Lagrangian curve germ $(L, 0) \subset\left(\mathbb{C}^{2}, 0\right)$ with an isolated singular point is $\mathcal{L}$-versal provided that the absolute KodairaSpencer map associated to $F$ is surjective.

Proof. We follow standard arguments due to Martinet for the case of singularity theory of differentiable maps [Mar82].

Put $f=F(0, \cdot)$ and let $G$ be an arbitrary $s$-parametric Lagrangian deformation of $f=F(0, \cdot)$. We have to prove that $G$ is $\mathcal{L}$-induced from $F$. To do this, we define the sum of $F$ and $G$ by the formula

$$
(F \oplus G)(\alpha, \lambda, x)=F(\lambda, x)+G(\alpha, x)-f .
$$

The restriction of this deformation to $\lambda=0$ is equal to $G$. Consequently, it is sufficient to prove that $F \oplus G$ is $\mathcal{L}$-induced from $F$. Denote by $F_{j}$ the restriction of $F \oplus G$ to the vector space $\left\{\alpha \mid \alpha_{k}=0 \forall k \geqslant j\right\}$. We have $F_{1}=F$ and $F_{s+1}=F \oplus G$.

The rigidity theorem implies that $F_{j}$ is $\mathcal{L}$-induced from $F_{j-1}$. By induction, we get that $F_{j}$ is $\mathcal{L}$-induced from $F_{j-k}$. In particular, $F_{s}=F \oplus G$ is $\mathcal{L}$-induced from $F_{1}=F$.

Remark 2. In the case $n>1$, we cannot use Martinet's argument since $F \oplus G$ is not, in general, a Lagrangian deformation.

Remark 3. For Lagrangian curves, there is a complete description of the $\mathcal{L}$-versal deformation. Let $F=f+\sum_{i=1}^{k} \lambda_{i} m_{i}$ be a $k$-parameter deformation of a reduced germ $\{f=0\}$. Denote by $e_{1}, \ldots, e_{\mu}$ the restrictions of the $m_{i}$ to $\lambda=0$. Then $F$ is $\mathcal{L}$-versal provided that the function germs $e_{1}, \ldots, e_{\mu} \in \mathcal{O}_{\mathbb{C}^{2}, 0}$ project to a basis $\left[e_{1}\right], \ldots,\left[e_{\mu}\right]$ of the $\mathbb{C}$-vector space $H^{1}\left(\mathcal{C}_{L, 0}^{\cdot}\right)$. If $f$ is quasihomogeneous, then according to Brieskorn [Bri70], there is a canonical isomorphism between the Milnor algebra and the fibre at the origin of the Brieskorn lattice of $f$. As the latter is isomorphic to the Lagrangian versal deformation space (cf. Example 2), we get an isomorphism

$$
H^{1}\left(\mathcal{C}_{L, 0}^{\bullet}\right) \longrightarrow \mathcal{O}_{\mathbb{C}^{2}, 0} / J f, \quad[m] \mapsto \bar{m} .
$$

Here $J f$ denotes the Jacobian ideal of $f$ generated by the partial derivatives of $f$. In this case, we recover the theorem of Colin de Verdière [Col03] similar (and somehow much more refined) results on volume forms are to be found in [Lan95, KL93] and reference therein.

Unlike the case of quasi-homogeneous singularities a set of germs $m_{1}, \ldots, m_{\mu}$ projecting to a basis of the Milnor algebra does not project to a basis of $H^{1}\left(\mathcal{C}_{L, 0}^{\bullet}\right) \approx \mathcal{O}_{L, 0} /\left\{\mathcal{O}_{L, 0}, f\right\}$. However, for a generic symplectic structure, this is indeed the case [VG82].

\section{Proof of the rigidity theorem}

\subsection{Infinitesimal formulation of the problem}

Let

$$
G:\left(\mathbb{C} \times \mathbb{C}^{k} \times \mathbb{C}^{2 n}, 0\right) \longrightarrow\left(\mathbb{C}^{n}, 0\right), \quad(t, \lambda, x) \mapsto G(t, \lambda, x)
$$

be a deformation of

$$
F:\left(\mathbb{C}^{k} \times \mathbb{C}^{2 n}, 0\right) \longrightarrow\left(\mathbb{C}^{n}, 0\right), \quad(\lambda, x) \mapsto F(\lambda, x) .
$$

Assertion. The deformation $G$ is $\mathcal{L}$-induced from $F$ provided that there exist function germs $h \in \mathcal{O}_{2 n+k+1}, b_{1}, \ldots, b_{k} \in \mathcal{O}_{k+1}$ and a matrix $B \in g l\left(n, \mathcal{O}_{2 n+k+1}\right)$ solving the equation

$$
\{h, G\}+B G+\sum_{i=1}^{k} b_{i} \partial_{\lambda_{i}} G=-\partial_{t} G .
$$


Proof. We search for a Poisson mapping germ

$$
\varphi:\left(\mathbb{C} \times \mathbb{C}^{k} \times \mathbb{C}^{2 n}, 0\right) \longrightarrow\left(\mathbb{C}^{2 n}, 0\right), \quad(t, \lambda, x) \longrightarrow \varphi(t, \lambda, x)
$$

and a matrix $A \in G L\left(n, \mathcal{O}_{2 n+k+1}\right)$ such that the following equalities hold

$$
\left\{\begin{array}{l}
F=A_{\tau}\left(G_{\tau} \circ \varphi_{\tau}\right), \\
\left(A_{0}, \varphi_{0}\right)=(I, I d) .
\end{array}\right.
$$

We have used the standard notations $I \in G L\left(n, \mathcal{O}_{2 n+k+1}\right)$ for the identity matrix, $I d$ for the identity mapping in $\mathbb{C}^{2 n}, A_{\tau}$ for $A(\tau, \cdot, \cdot)$ and so on.

Differentiating the first equation of the system (2) with respect to $\tau$ at $\tau=t$, we get the equation

$$
\left.A_{t} \frac{d}{d \tau}\right|_{\tau=t}\left(G_{t} \circ \varphi_{\tau}\right)+\left(\left.\frac{d}{d \tau}\right|_{\tau=t} A_{\tau}\right)\left(G_{t} \circ \varphi_{t}\right)+A_{t}\left(\left.\frac{d}{d \tau}\right|_{\tau=t} G_{\tau}\right) \circ \varphi_{t}=0 .
$$

Define the time-dependent vector field germ $v_{t}$ and the matrix $B \in g l\left(n, \mathcal{O}_{2 n+k+1}\right)$ by the formulae

$$
\begin{gathered}
v_{t}\left(\varphi_{t}(\lambda, x)\right)=\left.\frac{d}{d \tau}\right|_{\tau=t} \varphi_{\tau}(\lambda, x), \\
\left(A_{t} B_{t}\right) \circ \varphi_{t}=\left.\frac{d}{d \tau}\right|_{\tau=t} A_{\tau} .
\end{gathered}
$$

Multiplying (3) on the right by $\varphi_{t}^{-1}$ and on the left by $A_{t}^{-1}$, we get the equation

$$
L_{v_{t}} G_{t}+B_{t} G_{t}+\partial_{t} G_{t}=0 .
$$

Standard theorems on differential equations imply that $\left(\varphi_{\tau}, A_{\tau}\right)$ satisfying the system $(2)$ can be found provided that there exists $\left(v_{t}, B_{t}\right)$ satisfying (4). Up to here our arguments have been standard and hold for most of the versal deformation theorems. We now come to the specificity of our situation. The vector field $v(t, \cdot)=v_{t}$ comes from a Poisson mapping germ, therefore it is of the type

$$
v=\sum_{i=1}^{2 n} a_{i} \partial_{x_{i}}+\sum_{i=1}^{k} b_{i} \partial_{\lambda_{i}}, \quad a_{i} \in \mathcal{O}_{2 n+k+1}, \quad b_{i} \in \mathcal{O}_{k+1}
$$

where the vector field $w=\sum_{i=1}^{2 n} a_{i} \partial_{x_{i}}$ is the Hamiltonian field of some function germ $h \in \mathcal{O}_{2 n+k+1}$. Consequently, (4) can be written in the form

$$
\{h, G\}+B G+\sum_{i=1}^{k} b_{i} \partial_{\lambda_{i}} G=-\partial_{t} G
$$

This proves our assertion.

\subsection{Solving the infinitesimal equation}

We interpret Equation (1) in cohomological terms.

Let $\varphi: Z \longrightarrow \Lambda$ and $\varphi^{\prime}: Z^{\prime} \longrightarrow \Lambda^{\prime}$ be representatives for the germs $F$ and $G$. We have the following commutative diagram.

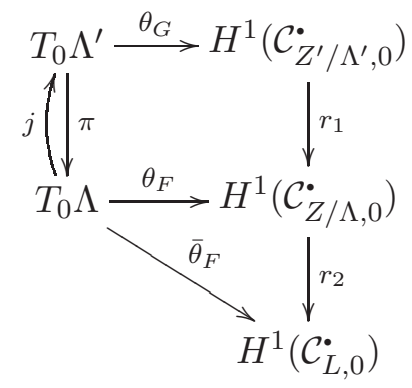




\section{A RIGIDITY THEOREM FOR LAGRANGIAN DEFORMATIONS}

The maps $\theta_{G}$ and $\theta_{F}$ are relative Kodaira-Spencer maps, while $\bar{\theta}_{F}$ is the absolute map. The maps $\pi, j$ denote, respectively, the canonical projection and the inclusion induced by the product structure $\left(\Lambda^{\prime}, 0\right) \approx(\Lambda \times \mathbb{C}, 0)$. The map $r_{1}$ is the restriction to $t=0$ and the map $r_{2}$ is the restriction to $\lambda=0$. In this setting, (1) can be rewritten as

$$
\sum_{i=1}^{k} b_{i} \theta_{G}\left(\partial_{\lambda_{i}}\right)=-\theta_{G}\left(\partial_{t}\right), \quad b_{1}, \ldots, b_{k} \in \mathcal{O}_{\Lambda^{\prime}, 0} .
$$

(We have used the notation $\theta_{G}\left(\partial_{\lambda_{i}}\right)$ rather than $\left[\partial_{\lambda_{i}} G\right]$ to underline in which space we take the cohomology class.)

Equation (6) can be solved provided that the cohomology class $\theta_{G}\left(\partial_{t}\right)$ belongs to the $\mathcal{O}_{\Lambda^{\prime}, 0^{-}}$ module generated by the $\theta_{G}\left(\partial_{\lambda_{i}}\right)$.

As $\bar{\theta}_{F}$ is surjective, we have an exact sequence (Remark $1, \S 1.4$ )

$$
0 \longrightarrow \mathcal{M} H^{1}\left(\mathcal{C}_{Z^{\prime} / \Lambda^{\prime}, 0}^{\cdot}\right) \stackrel{i}{\longrightarrow} H^{1}\left(\mathcal{C}_{Z^{\prime} / \Lambda^{\prime}, 0}^{\cdot}\right) \stackrel{r}{\longrightarrow} H^{1}\left(\mathcal{C}_{L, 0}^{\bullet}\right) \longrightarrow 0
$$

where $\mathcal{M}$ denotes the maximal ideal of the local $\operatorname{ring} \mathcal{O}_{\Lambda^{\prime}, 0}$.

The finiteness theorem (Theorem 2, $\S 1.5$ ) asserts that the $\mathcal{O}_{\Lambda^{\prime}, 0}$-module $H^{1}\left(\mathcal{C}_{Z^{\prime} / \Lambda^{\prime}, 0}^{\bullet}\right.$ ) is of finite type. Therefore, as the vectors

$$
\bar{\theta}_{F}\left(\partial_{\lambda_{1}}\right), \ldots, \bar{\theta}_{F}\left(\partial_{\lambda_{k}}\right)
$$

generate the vector space $H^{1}\left(\mathcal{C}_{L, 0}^{\bullet}\right)$, the Nakayama lemma implies that the elements

$$
\theta_{G}\left(\partial_{\lambda_{1}}\right), \ldots, \theta_{G}\left(\partial_{\lambda_{k}}\right)
$$

generate the $\mathcal{O}_{\Lambda^{\prime}, 0}$-module $H^{1}\left(\mathcal{C}_{Z^{\prime} / \Lambda^{\prime}, 0}^{\bullet}\right)$. This concludes the proof of Theorem 3.

\section{The coherence theorem}

For the proof of the rigidity theorem to be complete, we need to prove the finiteness of the module of relative infinitesimal Lagrangian deformations.

\subsection{Coherence of the higher direct image sheaves of the Lagrange complex}

We denote by $D_{r} \subset \mathbb{C}^{k+2 n}$ the polycylinder of polyradius $r \in \mathbb{C}^{k+2 n}$ centered at the origin.

Definition. A standard representative $\varphi: Z \longrightarrow \Lambda$ of a deformation germ $F:\left(\mathbb{C}^{k} \times \mathbb{C}^{2 n}, 0\right) \longrightarrow$ $\left(\mathbb{C}^{p}, 0\right)$ is a Stein representative of the germ together with a stratification of the fibres of $\varphi$ such that:

(1) $\varphi$ is the restriction to $Z=Y \cap D_{s}$ of a deformation of the type $\varphi^{\prime}: Y \longrightarrow \Lambda$, where $Y$ is a subvariety of $D_{r}, r>s$;

(2) there exists a fundamental system $\left(\Lambda_{t}\right)$ of neighbourhoods of the origin in $\mathbb{C}^{k}$ with $\Lambda_{1}=\Lambda$ such that for any $t \in] 0,1\left[\right.$, the fibres of $\varphi^{\prime}$ above $\Lambda_{t}$ are transverse to the boundary of $\bar{D}_{t s}$ (as stratified varieties).

There is a priori no reason for such a representative to exist.

Proposition 3. Any pyramidal Lagrangian deformation germ admits a standard representative.

Proof. First consider the case of a constant deformation $\varphi: L \mapsto\{0\}$. The stratification defined in $\S 1.5$ is obviously a Whitney stratification. In a Whitney stratification the transversality to a stratum (here the origin) implies the transversality to any adjacent strata in a small neighbourhood of it [Whi64, Tei81]. The existence of a standard representative follows. 


\section{D. GARAY}

Consider now a general deformation $F=\left(F_{1}, \ldots, F_{p}\right)$ of a Lagrangian germ $(L, 0)$. Choose a standard representative $\varphi: L \mapsto\{0\}, L \subset D_{s}$ of the constant deformation. That $\varphi$ is a standard representative implies that for $\lambda=0$, the vector space generated by hamiltonian vector fields of the $F_{i}(\lambda,-)$ at any point is transverse to the boundary of the polydisks $\left.\left.D_{t s}, t \in\right] 0,1\right]$. The transversality lemma implies that this remains true for $\lambda \in \mathbb{C}^{k}$ sufficiently close to the origin. This proves the proposition.

TheOREM 4. Let $\varphi: Z \longrightarrow \Lambda$ be a standard representative of a pyramidal Lagrangian deformation. Then, the direct image sheaves $\mathbb{R}^{p} \varphi_{*} \mathcal{C}_{Z / \Lambda}$ are coherent sheaves of $\mathcal{O}_{\Lambda}$ modules and there is a canonical isomorphism

$$
\left(\mathbb{R}^{p} \varphi_{*} \mathcal{C}_{Z / \Lambda}^{\cdot}\right)_{0} \approx H^{p}\left(\mathcal{C}_{Z / \Lambda, 0}^{\bullet}\right)
$$

\subsection{Criteria for the existence of a shrinking}

Let $\varphi: Z \longrightarrow \Lambda$ be a standard representative of a holomorphic map germ and denote by $\mathcal{K}^{\bullet}$ a complex of coherent sheaves with a $\varphi^{-1} \mathcal{O}_{\Lambda}$ linear differential. We use the notation of the above definition.

Definition [vSt]. A transversal vector field to $\left(\varphi, \mathcal{K}^{*}\right)$ is a $C^{\infty}$ vector field $\theta$ defined in $D_{t s} \backslash\{0\}$, $t>1$ satisfying the following conditions:

(1) for any $t \in] 0,1], \theta$ is transversal to the boundary of $D_{t s}$ above $\Lambda_{t}$;

(2) the integral curves of $\theta$ are contained in the fibres of $\varphi$;

(3) the restriction of the cohomology sheaves $\mathcal{H}^{p}\left(\mathcal{K}^{*}\right)$ to the integral curves of $\theta$ are constant sheaves.

The following theorem is a consequence of the Kiehl-Verdier theorem [KV71, Dou74] (see also [FK71]).

Theorem 5 [vSt]. The existence of a transversal vector field to $\left(\varphi, \mathcal{K}^{*}\right)$ implies that the hypercohomology sheaves $\mathbb{R}^{p} f_{*} \mathcal{K}^{\bullet}$ are coherent and that there is a canonical isomorphism

$$
\left(\mathbb{R}^{p} \varphi_{*} \mathcal{K}\right)_{0} \approx H^{p}\left(\mathcal{K}_{0}^{\bullet}\right)
$$

Remark 4. This theorem is proved only in the case $\operatorname{dim} S=1$ and $X$ is smooth in [vSt]. These assumptions are nevertheless not used in the proof.

\subsection{Proof of the coherence theorem}

We construct a transversal vector field $\theta$ to $\left(\varphi, \mathcal{C}_{Z / \Lambda}^{\bullet}\right)$. The fact that $\varphi$ is a standard representative implies that at any point $x \in \partial \bar{D}_{t s}$ there exists a holomorphic function $h \in \mathcal{I}_{x}$ such that the Hamilton vector field $X$ of $h$ is transverse to $\partial \bar{D}_{t s}$ and points inward $D_{t s}$, provided that $\varphi(x) \in \Lambda_{t}$.

Proposition 2 implies that the cohomology sheaves $\mathcal{H}^{p}\left(\mathcal{C}_{Z / \Lambda}^{\bullet}\right)$ are constant along the integral lines of $X$. Consequently, for $t \in] 0,1]$, we can find a covering $\left(V_{k}\right)$ of $D_{s} \backslash\{0\}$ such that there exists a Hamiltonian vector field $X_{k}$ in $V_{k}$ transversal to $\left(\varphi, \mathcal{C}_{Z / \Lambda}^{*}\right)$.

Take a partition of the unity $\left(\psi_{k}, V_{k}\right)$ of $D_{s} \backslash\{0\}$ and define the $C^{\infty}$ vector-field

$$
\theta=\sum_{k=1}^{s} \psi_{k} X_{k} .
$$

This vector field is transversal to $\left(\varphi, \mathcal{C}_{Z / \Lambda}^{\bullet}\right)$. This concludes the proof of Theorem 4. 


\section{A RIGIDITY THEOREM FOR LAGRANGIAN DEFORMATIONS}

\section{ACKNowledGements}

The author thanks the Deutsche Forschungsgemeinschaft for financial support. It is a pleasure to thank D. van Straten for numerous discussions from which this work originated and C. Sevenheck for his explanations on singular Lagrangian varieties. The author also acknowledges G. Capitanio, M. Chaperon, P. Dingoyan, H. Eliasson E. Ferrand, B. Malgrange, D. Mond, B. Teissier, C. Voisin as well as the anonymous referee for pertinent comments and remarks on the preliminary version of this text. Last, but not least, the author thanks Y. Colin de Verdière whose work and questions motivated the present research.

\section{REFERENCES}

Arn81 V. I. Arnold, Lagrangian manifolds with singularities, asymptotic rays and the unfurled swallowtail, Funct. Anal. Appl. 15 (4) (1981), 1-14.

Bri70 E. Brieskorn, Die Monodromie der isolierten Singularitäten von Hyperflächen, Manuscripta Math. 2 (1970), 103-161.

Col03 Y. Colin de Verdière, Singular Lagrangian manifolds and semi-classical analysis, Duke Math. J. 116 (2003), 263-298.

CP94 Y. Colin de Verdière and B. Parisse, Équilibre instable en régime semi-classique I: concentration micro locale, Comm. Partial Differential Equations 19 (1994), 1535-1564.

CP99 Y. Colin de Verdière and B. Parisse, Singular Bohr-Sommerfeld rules, Comm. Math. Phys. 205 (1999), 459-500.

Dou74 A. Douady, Le théorème des images directes de Grauert (d'après Kiehl-Verdier), Astérisque 16 (1974), 49-62.

DS99 M. Dimassi and J. Sjöstrand, Spectral asymptotics in the semi-classical limit, London Mathematical Society Lecture Note Series, no. 268 (Cambridge University Press, Cambridge, 1999).

FK71 O. Forster and K. Knorr, Ein Beweis des Grauertschen Bildgarbensatzes nach Ideen von B. Malgrange, Manuscripta Math. 5 (1971), 19-44.

Gar02 M. D. Garay, Stable complete integrability and singular Lagrangian varieties, Preprint (2002), Mainz Universität.

Gar04 M. D. Garay, An isochore versal deformation theorem, Topology 43 (2004), 1081-1088.

Gar05a M. D. Garay, Stable momentum mappings and singular Lagrangian foliations, Quart. J. Math. 56 (2005), 357-366.

Gar05b M. D. Garay, Analytic quantum mechanics, Preprint (2005), arXiv:math-ph/0502027.

Giv90 A. B. Givental, Singular Lagrangian manifolds and their Lagrangian mappings, J. Soviet Math. 52 (1990), 3246-3278.

Gre75 G.-M. Greuel, Der Gauss-Manin-Zusammenhang isolierter Singularitäten von vollständigen Durchschnitten, Math. Ann. 214 (1975), 235-266.

GS03 M. D. Garay and D. van Straten, On the topology of Lagrangian Milnor fibres, Internat. Math. Res. Notices 35 (2003), 1933-1943.

KL93 V. P Kostov and S. K Lando, Versal deformations of powers of volume forms, in Computational algebraic geometry (Nice, 1992), Progress in Mathematics, vol. 109 (Birkhäuser, Basel, 1993), 143-162.

KV71 R. Kiehl and J. L. Verdier, Ein Einfacher Beweis des Kohärenzsatzes von Grauert, Math. Ann. 195 (1971), 24-50.

Lan95 S. K. Lando, Deformations of differential forms, Trudy Mat. Inst. Steklov 209 (1995), 167-199.

Mar82 J. Martinet, Singularities of smooth functions and maps, London Mathematical Society Lecture Note Series, vol. 58 (Cambridge University Press, Cambridge, 1982).

NP91 H. D. Nguyen and F. Pham, Germes de configurations legendriennes stables et fonctions d'AiryWeber généralisées, Ann. Inst. Fourier (Grenoble) 41 (1991), 905-936. 


\section{A RIGIDITY THEOREM FOR LAGRANGIAN DEFORMATIONS}

Pha00 F. Pham, Multiple turning points in exact WKB analysis (variations on a theme of Stokes), in Toward the exact WKB analysis of differential equations, linear or non-linear, eds C. Howls, T. Kawai and Y. Takei (Kyoto University Press, Kyoto, 2000), 71-85.

Pou74 G. Pourcin, Déformation de singularités isolées, Astérisque 16 (1974), 161-173.

Rus64 H. Rüssmann, Über das Verhalten analytischer Hamiltonscher Differentialgleichungen in der Nühe einer Gleichgewichtslösung, Math. Ann. 154 (1964), 285-306.

Sev03 C. Sevenheck, Lagrange-Singularitäten, PhD thesis, J. Gutemberg Universität, Mainz, 2003 (in German).

SvS03 C. Sevenheck and D. van Straten, Deformation of singular Lagrangian subvarieties, Math. Ann. 327 (2003), 79-102.

Tei81 B. Teissier, Variétés polaires II, in Algebraic Geometry Proc. (La Mábida), Lecture Notes in Mathematics, vol. 961 (Springer, Berlin, 1981), 314-495.

vSt D. van Straten, On the Betti numbers of the Milnor fibre of a certain class of hypersurface singularities, in Singularities, representation of algebras and vector bundles, eds G.-M. Greuel and G. Trautmann, Lecture Notes in Mathematics, vol. 1273 (Springer, Berlin, 1987), 203-220.

Vey78 J. Vey, Sur certains systèmes dynamiques séparables, Amer. J. Math. 100 (1978), 591-614.

VG82 A. B. Varchenko and A. N. Givental, The period mapping and the intersection form, Funct. Anal. Appl. 16 (1982), 83-93.

Voi92 C. Voisin, Sur la stabilité des sous-variétés lagrangiennes des variétés symplectiques holomorphes, in Complex projective geometry, eds G. Ellingsrud, C. Peskine, G. Sacchiero and S. A. Stromme, London Mathematical Society Lecture Note Series, vol. 179 (Cambridge University Press, Cambridge, 1992), 294-302.

Wei73 A. Weinstein, Lagrangian submanifolds and Hamiltonian systems, Ann. of Math. (2) 98 (1973), 377-410.

Whi64 H. Whitney, Tangents to an analytic variety, Ann. of Math. (2) 81 (1964), 496-549.

Mauricio D. Garay garay@mathematik.uni-mainz.de

Fachbereich Mathematik 17, Staudingerweg 9, Johannes Gutenberg-Universität, 55099 Mainz, Germany 